functions as a stress-responsive regulator of angiogenesis in the murine heart. J. Clin. Invest. 117:3198-3210. doi:10.1172/JCI32573.

13. Oka, T., Xu, J., and Molkentin, J.D. 2007. Reemployment of developmental transcription factors in adult heart disease. Semin. Cell Dev. Biol. 18:117-131.

14. Morisco, C., et al. 2001. Glycogen synthase kinase 3 beta regulates GATA4 in cardiac myocytes. J. Biol. Chem. 276:28586-28597.

15. Charron, F., et al. 2001. Tissue-specific GATA factors are transcriptional effectors of the small GTPase RhoA. Genes Dev. 15:2702-2719.

16. Liang, Q., et al. 2001. The transcription factor GATA4 is activated by extracellular signal-regulated kinase 1- and 2-mediated phosphorylation of serine 105 in cardiomyocytes. Mol. Cell. Biol. 21:7460-7469.

17. Brugarolas, J.B., Vazquez, F., Reddy, A., Sellers, W.R., and Kaelin, W.G., Jr. 2003. TSC2 regulates
VEGF through mTOR-dependent and -independent pathways. Cancer Cell. 4:147-158.

18. Takahashi, A., et al. 2002. Myogenic Akt signaling regulates blood vessel recruitment during myofiber growth. Mol. Cell. Biol 22:4803-4814.

19. Tirziu, D., et al. 2007. Myocardial hypertrophy in the absence of external stimuli is induced by angiogenesis in mice. J. Clin. Invest. 117:3188-3197. doi:10.1172/JCI32024.

20. Carmeliet, P., et al. 1999. Impaired myocardial angiogenesis and ischemic cardiomyopathy in mice lacking the vascular endothelial growth factor isoforms VEGF164 and VEGF188. Nat. Med. 5:495-502.

21. Giordano, F.J., et al. 2001. A cardiac myocyte vascular endothelial growth factor paracrine pathway is required to maintain cardiac function. Proc. Natl. Acad. Sci. U. S. A. 98:5780-5785.

22. Dor, Y., et al. 2002. Conditional switching of VEGF provides new insights into adult neovas- cularization and pro-angiogenic therapy. EMBOJ. 21:1939-1947.

23. Folkman, J. 1998. Is tissue mass regulated by vascular endothelial cells? Prostate as the first evidence. Endocrinology. 139:441-442.

24. Folkman, J. 2001. Angiogenesis. In Harrison's principles of internal medicine. 15th edition. E. Braunwald, et al., editors. McGraw-Hill. New York, New York, USA. 517-530.

25. Rupnick, M.A., et al. 2002. Adipose tissue mass can be regulated through the vasculature. Proc. Natl. Acad. Sci. U. S. A. 99:10730-10735.

26. Franck-Lissbrant, I., Haggstrom, S., Damber, J.E., and Bergh, A. 1998. Testosterone stimulates angiogenesis and vascular regrowth in the ventral prostate in castrated adult rats. Endocrinology. 139:451-456.

27. LeCouter, J., et al. 2003. Angiogenesis-independent endothelial protection of liver: role of VEGFR-1. Science. 299:890-893.

\title{
Kidney kinase network regulates renal ion cotransport
}

\author{
Nati Hernando, Carsten Wagner, Jürg Biber, and Heini Murer
}

Institute of Physiology, University of Zurich, Zurich, Switzerland.

\begin{abstract}
Protein kinases catalyze the phosphorylation of serine/threonine or tyrosine residues, which may directly alter a protein's functional properties. Kinases can also regulate protein functions indirectly, for example, by controlling the composition and/or subcellular localization of members of multiprotein complexes that associate with the regulated protein. In this issue of the JCI, two separate studies by Weinman et al. and Yang et al. examine the second of these two modes of kinase-mediated regulation and demonstrate the effects of kinases on two $\mathrm{Na}^{+}$-driven renal cotransporters (see the related articles beginning on pages 3403 and 3412). Their results reveal important implications for phosphate and salt homeostasis, respectively.
\end{abstract}

Homeostatic control of body fluids is achieved by, among other mechanisms, a variety of solute transporters that operate according to the body's needs. Thus, the function of these transporters is modulated by diverse regulatory systems. Frequently, the final event in a hormonally stimulated signaling cascade involves activation of protein kinases that modify transporter activities by changing the characteristics or the subcellular localization of the transporters. Such alterations

Nonstandard abbreviations used: BBM, brush border membrane; DCT, distal convoluted tubule; NCC, thiazide-sensitive $\mathrm{Na}-\mathrm{Cl}$ cotransporter; NHERF-1, sodiumhydrogen exchanger regulatory factor- $1 ; \mathrm{Npt} 2 \mathrm{a}, \mathrm{Na}^{+}-\mathrm{P}_{\mathrm{i}}$

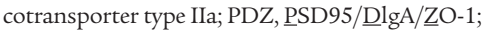
$\mathrm{P}_{\mathrm{i}}$, inorganic phosphate; $\mathrm{PT}$, proximal tubule; $\mathrm{PTH}$, parathyroid hormone; WNK, with no lysine ( $\underline{\mathrm{K}})$.

Conflict of interest: The authors have declared that no conflict of interest exists.

Citation for this article: J. Clin. Invest. 117:3179-3182 (2007). doi:10.1172/JCI33859. can be achieved by direct phosphorylation of the transporter itself or via phosphorylation of proteins that are part of heteromultimeric complexes.

Protein kinase-mediated activation or inhibition of many hormonally controlled transporters have been described, yet the final targets in these signaling pathways and/or the underlying regulatory mechanisms have not yet been identified. The work presented in this issue of the JCI in the articles by Weinman et al. (1) and Yang et al. (2) now sheds some mechanistic light on how the activity and/or abundance of ion transporters are hormonally controlled by protein complexes that contain protein kinases. These two reports respectively address the regulation of two ion transporters that are predominantly expressed in kidney: the parathyroid hormone-regulated (PTH-regulated) $\mathrm{Na}^{+}-\mathrm{P}_{\mathrm{i}}$ cotransporter type IIa (Npt2a; encoded by SLC34A1 and also known as NaPi-IIa) (3) and the thiazide-sensitive $\mathrm{Na}-\mathrm{Cl}$ cotransporter (NCC; encoded by SLC12A3 and also known as TSC) (4). The NCC is located on the luminal side of distal convoluted tubule (DCT) cells, whereas Npt2a associates with the brush border membranes (BBMs) of proximal tubules (PTs) (3, 5). Apical expression of NCC and Npt2a is under the control of kinases such as WNK kinases (with no lysine $[\underline{K}]$ in their catalytic domain II) and PKA/PKC, respectively. Interestingly, neither WNK nor PKA/PKC have been reported to phosphorylate these transporters directly $(3,6-8)$.

\section{Npt2a and NCC: function and dysfunction}

Npt2a mediates $\mathrm{Na}^{+}$-coupled, electrogenic reabsorption of inorganic phosphate $\left(\mathrm{P}_{\mathrm{i}}\right)$. The abundance of the Npt2a protein is regulated by dietary phosphate intake, by phosphaturic PTH, and by different phosphatonins $(3,8,9)$. The importance of Npt2a in phosphate homeostasis was documented in an $\mathrm{Npt2}^{-/-}$mouse model that is characterized by hypophosphatemia (abnormally decreased level of phosphate in the blood) and hyperphosphaturia (increased excretion of phosphate in the urine) due to reduced $\mathrm{P}_{\mathrm{i}}$ reabsorption (10). Npt2a expression is reduced in animal models of X-linked hypophosphatemia, and a similar defect may also be implicated 


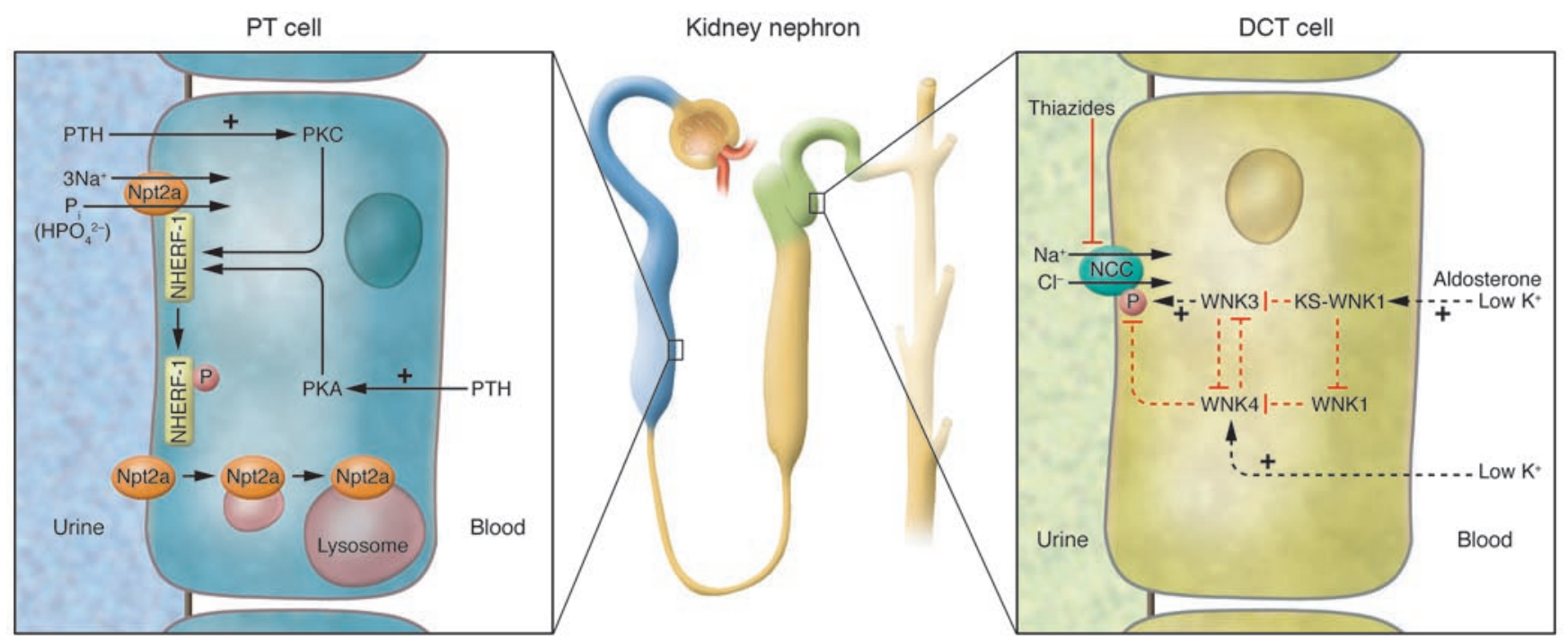

\section{Figure 1}

Kinase signaling networks regulating ion transport in the kidney. Left: Regulation of the PT cell Na+- $\mathrm{P}_{\mathrm{i}}$ cotransporter Npt2a by PTH, via apical and basolateral PTH receptors. As reported in this issue by Weinman et al. (1), phosphorylation at Ser77 of NHERF-1 results in a dissociation of Npt2a from NHERF-1, consequent Npt2a internalization, and an increased excretion of phosphate in the urine. Right: DCT cells express the $\mathrm{Na}^{+}-\mathrm{Cl}^{-}$cotransporter NCC, which is inhibited by thiazides. The study by Yang et al. (2) in this issue of the $\mathrm{JCl}$ reports that WNK3, a member of the WNK kinase family, interacts with WNK1 and WNK4 to regulate the phosphorylation and activity of NCC. WNK kinases are regulated by stimuli such as changes in aldosterone or extracellular potassium levels. Dotted arrows indicate that the nature of interaction between kinases (direct or indirect) is not known to date. KS-WNK1, kidney-specific WNK1.

in human hypophosphatemic syndromes (9). However, Npt2a downregulation is secondary to alterations in different regulatory factors (e.g. FGF23, one of the so-called phosphatonins) $(9,11)$.

NCC belongs to the SLC12 electroneutral cation-Cl-coupled cotransporter family (4), mediates reabsorption of $\mathrm{NaCl}$, and is the target of widely used thiazide diuretics, which due to their vasodilator properties are often used to treat hypertension. Inactivating mutations in NCC cause Gitelman disease, an autosomal recessive salt-wasting $\left(\mathrm{NaCl}, \mathrm{K}^{+}, \mathrm{Mg}^{2+}\right)$ disorder characterized by hypotension $(4,5)$. Autosomal dominant Gordon disease, a consequence of increased NCC activity, is characterized by hypertension, hyperkalemia, and hyperchloremic metabolic acidosis. The NCC overactivity is due to increased expression of NCC in the apical membrane and hypertrophy of the DCT, secondary to mutations in the regulatory WNK1 and/or WNK4 kinases $(4,5,6,12)$.

\section{Npt2a regulation by kinases and protein networks}

PTH binds to its $G$ protein-coupled receptor in the $\mathrm{PT}$ and, through activation of PKA and PKC, modulates the apical abundance of Npt2a by controlling the rate of endocytotic Npt2a retrieval
(Figure 1, left). PTH-induced Npt2a internalization does not involve phosphorylation of the cotransporter itself; therefore, it has been postulated that PTH-induced kinase activity indirectly affects the positioning of the Npt2a protein within the $\operatorname{BBM}(8,13)$.

A major issue in protein kinase signaling is how these enzymes achieve their spatial-substrate specificity. More than 30 A kinase-anchoring proteins (AKAPs) and several receptors for activated kinase C (RACKs), with different tissue distribution and subcellular locations, are responsible for subcellular compartmentalization of protein kinases and phosphatases $(14,15)$. These anchoring proteins are part of heteromultimeric protein networks that place the kinases and/or phosphatases in close proximity to their substrates. In the case of Npt2a, such networks are built around the sodiumhydrogen exchanger regulatory factor-1 (NHERF-1) family $(16,17)$.

NHERF-1 was identified as a cofactor required for PKA-mediated inhibition of the $\mathrm{Na}^{+} / \mathrm{H}^{+}$exchanger NHE3, due to its ability to simultaneously bind to NHE3 and ezrin, an AKAP expressed in epithelia (for review, see ref. 16). The NHERF family consists of 4 related proteins that contain either 2 (NHERF-1/2) or 4 (NHERF-3/4)
PSD95/미A/ZO-1 (PDZ) domains for protein-protein interaction. NHERF-1/2 can also bind to several other transporters, G protein-coupled receptors (including the PTH receptor), and phospholipase C (PLC) (18). PDZ-based interactions can be regulated by phosphorylation of either protein partner involved in the protein-protein interaction. Several kinases, including cell division cycle 2 kinase and G proteincoupled receptor kinase 6, phosphorylate NHERF-1 in vitro (19).

Npt2a interacts via its $C$ terminus with several PDZ-containing proteins located in or in close proximity to the BBM, including the 4 members of the NHERF family (20). Npt2a binds to PDZ1 of NHERF-1/2 and to PDZ3 of NHERF3/4. The last 3 amino acids of Npt2a are critical for these interactions. Npt2a expression is only minimally impacted in NHERF3-deficient mice. However NHERF-1 deficiency is manifested by a reduced expression of Npt2a in the BBM and by an impaired regulation by $\mathrm{PTH}$ via the $\mathrm{PKC}$ pathway $(17,21,22)$. This suggests an important role of NHERF-1 in the apical stabilization of Npt2a and in scaffolding components of the PKC signaling pathway.

The abundance and subcellular localization of NHERF-1, in contrast to Npt2a is not affected by PTH, suggesting that 
an alternative mechanism is necessary for the dissociation of the Npt2a/PDZ complex. In their study in this issue of the JCI, Weinman and coworkers show that in renal PT, PKA and PKC phosphorylate NHERF-1 on Ser77, a residue located within the first PDZ domain (1) (Figure 1, left). From studies performed with renal PT cells derived from NHERF-1-deficient mice and infected with several adenovirus-NHERF-1 constructs, the authors conclude that phosphorylation of Ser77 is responsible for the PTH-induced dissociation of the NHERF-1/Npt2a complex, allowing for the retrieval of the Npt2a cotransporter from the BBM, whereas NHERF-1 remains attached to the apical cytoskeleton (Figure 1, left). Thus, phosphorylation of Ser77 of NHERF-1 may represent the long-sought "off switch" that determines the lifespan of Npt2a within the apical membrane, whereby the off signal could be a conformational change within the PDZ1 domain of NHERF-1. Interestingly, the authors report that the mechanisms of phosphorylation of Ser77 by PKC and PKA differ. Whereas PKC appears to directly phosphorylate NHERF-1 at position Ser77, PKA-mediated NHERF-1 phosphorylation seems to require a phosphatase. This latter finding indicates the presence of a rather complex phosphorylation machinery within the apical heteromultimeric network that contains Npt2a. Future work will undoubtedly uncover the full composition of this network and thereby help to unravel other mechanisms involved in the regulation of $\mathrm{Npt} 2 \mathrm{a}$, such as those involving cGMP and MAPK kinases and phosphatonins $(8,9,11)$.

\section{NCC regulation by kinase networks}

NCC activity is directly associated with regulation of blood pressure and thus must be tightly controlled. Analysis of the underlying mechanisms leading to Gordon syndrome (pseudohypoaldosteronism type II) offers insights into the regulation of NCC and blood pressure. Hypertension, a symptom of Gordon syndrome, is very sensitive to treatment with thiazide diuretics (12), suggesting an important role of NCC as part of the pathomechanism. Gordon syndrome is caused by mutations in the kinases WNK1 and WNK4 (21). Four members of the WNK kinase family are known in humans, and several studies in the past years have uncovered an important role for these kinases in the regulation of epithelial cell transport of potassium, sodium, and chloride via the regulation of several ion channels (e.g., renal outer medullary potassium channel [ROMK], epithelial sodium channel [ENaC], and claudins) and electrolyte transporters $\left(\mathrm{Na}^{+}-\mathrm{K}^{+}-2 \mathrm{Cl}\right.$ cotransporter 1 [NKCC1], NKCC2, NCC, $\mathrm{K}^{+}-\mathrm{Cl}^{-}$cotransporters $1-4$ [KCC1-4]) (23). The emerging picture indicates that WNK kinases may interact with each other and with other kinases such as serum/glucocorticoid-regulated kinase 1 (SGK1) to directly and indirectly phosphorylate target proteins and thereby alter their activity. The stimulatory effect of WNK3 and the inhibitory effect of WNK4 on NCC activity had been shown $(7,24)$, but the underlying mechanisms of these effects as well as that of the interplay of WNK kinases with other kinases remained elusive. In this issue of the JCI, Yang et al. (2) analyze the role of WNK3 in the network of NCC regulation, which also involves WNK1 and WNK4, using heterologous expression in Xenopus oocytes. The major finding is that WNK1, as well as the short, kidney-specific isoform of WNK1 known as KS-WNK1, WNK3, and WNK4 form a regulatory complex of physically interacting and mutually regulating kinases (Figure 1, right). These interactions do not always require the kinase-active domains, suggesting that in some instances WNK kinases may also act as scaffolding proteins for other (WNK) kinases. More interestingly, the effect of coexpression of WNK kinase isoforms with NCC showed a graded response depending on the actual stoichiometry of kinases present. Thus, WNK kinases are not providing a simple on-off switch; instead, the data imply that the presence of several WNK kinase isoforms that regulate each other in the renal DCT allows for the fine tuning of NCC over a wide range of transport activity. Expression of WNK kinase isoforms is influenced by factors such a dietary potassium intake or aldosterone and may therefore integrate these inputs into the differential regulation of transport mechanisms for potassium and $\mathrm{NaCl}$. In addition, Yang et al. also offer a new interpretation of the pathomechanism of Gordon syndrome. Mutations in WNK4 (i.e. WNK4 Q562E) cause hypertension by disinhibiting NCC activity, a trait inherited in an autosomal dominant manner (25), which cannot be easily reconciled with the direct inhibitory effect of WNK4 on NCC activity. In their current study, Yang et al. (2) show that WNK4 Q562E acts as a dominant-negative inhibitor of normal WNK4 function and thereby releases the inhibition of NCC, causing hyperactivity of this important transporter.

\section{Conclusions}

The studies by Weinman et al. (1) and Yang et al. (2) described here provide compelling evidence for the emerging view that the regulation of membrane transporter function is not a linear cascade of events but the result of the integration of various signals that requires a complex machinery of receptors, kinases, phosphatases, and scaffolding proteins and allows for the graded and fine-tuned adaptation of transporter function to cellular or whole-body needs. We are only just beginning to understand how such macromolecular complexes are built and regulated.

\section{Acknowledgments}

The authors are supported by grants 31065397 (to H. Murer) and 31-109677 (to C.A. Wagner) of the Swiss National Fonds and the EuReGene project of the 6th European Union framework program to H. Murer and C.A. Wagner.

Address correspondence to: Jürg Biber, Institute of Physiology, University of Zurich, Winterthurerstrasse 190, CH-8057 Zurich, Switzerland. Phone: 41-044-6355032; Fax: 41-044-635-5715; E-mail: JuergBiber@access.uzh.ch.

1. Weinman, E.J., et al. 2007. Parathyroid hormone inhibits renal phosphate transport by phosphorylation of serine 77 of sodium-hydrogen exchanger regulatory factor-1. J. Clin. Invest. 117:3412-3420. doi:10.1172/JCI32738.

2. Yang, C.-L., Zhu, X., and Ellison, D.H. 2007. The thiazide-sensitive $\mathrm{Na}-\mathrm{Cl}$ cotransporter is regulated by a WNK kinase signaling complex. J. Clin. Invest. 117:3403-3411. doi:10.1172/JCI32033.

3. Murer, H., Forster, I., and Biber, J. 2004. The sodium phosphate cotransporter family SLC34. Pflugers Arch. 447:763-767.

4. Hebert, S.C., Mount, D.B., and Gamba, G. 2004. Molecular physiology of cation-coupled Clcotransport: the SLC12 family. Pflugers Arch. 447:580-593.

5. Gamba, G. 2005. Molecular physiology and pathophysiology of electroneutral cation-chloride cotransporters. Physiol. Rev. 85:423-493.

6. Gamba, G. 2005. Role of WNK kinases in regulating tubular salt and potassium transport and in the development of hypertension. Am. J. Physiol. Renal Physiol. 288:F245-F252.

7. Yang, C.L., Zhu, X., Wang, Z., Subramanya, A.R., and Ellison, D.H. 2005. Mechanisms of WNK1 and WNK4 interaction in the regulation of thiazide-sen- 
sitive $\mathrm{NaCl}$ cotransport. J. Clin. Invest. 115:1379-1387. doi:10.1172/JCI200522452.

8. Forster, I.C., Hernando, N., Biber, J., and Murer, H. 2006. Proximal tubular handling of phosphate: a molecular perspective. Kidney Int 70:1548-1559

9. Tenenhouse, H.S. 2007. Phosphate transport: Molecular basis, regulation and pathophysiology. J. Steroid Biochem. Mol. Biol. 103:572-577.

10. Beck, L., et al. 1998. Targeted inactivation of Npt2 in mice leads to severe renal phosphate wasting, hypercalciuria, and skeletal abnormalities. Proc. Natl. Acad. Sci. U. S. A. 95:5372-5327.

11. Berndt, T., and Kumar, R. 2007. Phosphatonins and the regulation of phosphate homeostasis. Annu. Rev. Physiol. 69:341-359.

12. Lalioti, M.D., et al. 2006. Wnk4 controls blood pressure and potassium homeostasis via regulation of mass and activity of the distal convoluted tubule. Nat. Genet. 38:1124-1132.

13. Deliot, N., et al. 2005. Parathyroid hormone treatment induces dissociation of type lla $\mathrm{Na}$ Pi cotransporter- $\mathrm{Na} / \mathrm{H}$ exchanger regulatory factor-1 complexes. Am. J. Physiol. Cell Physiol. 289:C159-C167.
14. Schechtman, D., and Mochly-Rosen, D. 2001. Adaptor proteins in protein kinase C-mediated signal transduction. Oncogene. 20:6339-6347.

15. Tasken, K., and Aandahl, E.M. 2004. Localized effects of cAMP mediated by distinct routes of protein kinase A. Physiol. Rev. 84:137-167.

16. Shenolikar, S., Voltz, J.W., Cunningham, R., and Weinman, E.J. 2004. Regulation of ion transport by the NHERF family of PDZ proteins. Physiology. 19:362-369.

17. Weinman, E.J., Cunningham, R., Wade, J.B., and Shenolikar, S. 2005. The role of NHERF-1 in the regulation of renal proximal tubule sodium-hydrogen exchanger 3 and sodium-dependent phosphate cotransporter 2a. J. Physiol. 567:27-32.

18. Weinman, E.J., Hall, R.A., Friedman, P.A., Liu-Chen, L.-Y., and Shenolikar, S. 2006. The association of NHERF adaptor proteins with $G$ protein-coupled receptors and receptor tyrosine kinases. Ann. Rev. Physiol. 68:491-505.

19. He, J., Lau, A.G., Yaffe, M.B., and Hall, R.A. 2001 Phosphorylation and cell cycle-dependent regulation of $\mathrm{Na} / \mathrm{H}$ exchanger regulatory factor- 1 by Cdc2 kinase. J. Biol. Chem. 276:41559-41565

20. Gisler, S.M., et al. 2001. Interaction of the type IIa
$\mathrm{Na} / \mathrm{Pi}$ cotransporter with PDZ proteins. J. Biol. Chem. 276:9206-9213.

21. Shenolikar, S., Voltz, J.W., Minkoff, C.M., Wade, J.B., and Weinman, E.J. 2002. Targeted disruption of the mouse NHERF-1 gene promotes internalization of proximal tubule sodium-phosphate cotransporter type IIa and renal phosphate wasting. Proc. Natl. Acad. Sci. U. S. A. 99:11470-11475.

22. Capuano, P., et al. 2007. Defective coupling of apical PTH receptors to phospholipase C prevents internalization of the $\mathrm{Na}^{+}$-phosphate cotransporter NaPi-IIa in Nherf1-deficient mice. Am. J. Physiol. Cell Pbysiol. 292:C927-C934.

23. Wilson, F.H., et al. 2001. Human hypertension caused by mutations in WNK kinases. Science. 293:1107-1112

24. Kahle, K.T., et al. 2006. WNK protein kinases modulate cellular Cl- flux by altering the phosphorylation state of the $\mathrm{Na}-\mathrm{K}-\mathrm{Cl}$ and $\mathrm{K}-\mathrm{Cl}$ cotransporters. Physiology. 21:326-335.

25. Rinehart, J., et al. 2005. WNK3 kinase is a positive regulator of NKCC2 and NCC, renal cation$\mathrm{Cl}$ - cotransporters required for normal blood pressure homeostasis. Proc. Natl. Acad. Sci. U. S. A. 102:16777-16782.

\title{
Macrophages feel their age in macular degeneration
}

\author{
Martine J. Jager ${ }^{1}$ and Caroline C.W. Klaver ${ }^{2,3}$
}

1Department of Ophthalmology, Leiden University Medical Center, Leiden, The Netherlands. 2Department of Ophthalmology and Department of Epidemiology and Biostatistics, Erasmus Medical Center, Rotterdam, The Netherlands. ${ }^{3}$ Netherlands Institute for Neuroscience, Amsterdam, The Netherlands.

\begin{abstract}
Macular degeneration, during which the posterior part of the eye known as the macula suffers from thinning, atrophy, and bleeding caused by abnormal angiogenesis (blood vessel formation), predominantly affects elderly adults and results in the loss of central vision. In this issue of the $J C I$, Kelly et al. investigate the regulation of innate immune cells, specifically macrophages, in ocular neovascularization following eye injury in mice (see the related article beginning on page 3421). They found that, as the mice aged, increased expression of IL-10 by senescent macrophages and changes in their expression of other cytokines altered the ability of these cells to restrain trauma-induced angiogenesis in the eye. These data provide insight into the effect of senescence on macrophage function and angiogenesis and have important implications for age-related diseases such as macular degeneration.
\end{abstract}

\section{Neovascularization and the eye}

The eye is a delicate structure with a finely developed blood vessel network, and any disturbances to this delicate system may lead to new blood vessel formation (angiogenesis) in areas of the eye that normally do not contain blood vessels, which may interfere with the function of the eye. In

Nonstandard abbreviations used: AMD, age-related macular degeneration; FasL, Fas ligand.

Conflict of interest: The authors have declared that no conflict of interest exists.

Citation for this article: J. Clin. Invest. 117:3182-3184 (2007). doi:10.1172/JCI34070. ocular diseases, abnormal blood vessel formation (neovascularization) often has a detrimental effect on vision. An important example of a disease in which the formation of abnormal blood vessels affects the eye is diabetic retinopathy, in which closure of retinal capillaries leads to ischemia, followed by the production of the proangiogenic growth factor VEGF, which induces the development of new blood vessels of an inferior type (1). Such vessels tend to bleed or grow into locations where they damage vision. Another example is age-related macular degeneration (AMD). While the central area of the retina, the macula, is normally devoid of blood vessels, in AMD, aberrant blood vessels develop in this area and destroy central vision. In response to hypoxia, these blood vessels grow from the underlying choroid through Bruch's membrane into the subretinal space (Figure 1). This type of AMD is known as neovascular or wet AMD; together with geographic atrophy and dry AMD (a discrete, central area with depigmentation and breakdown of light-sensitive cells in the macula), these are the clinical forms known as AMD. Advanced AMD is a frequent cause of severe visual impairment among the elderly. The estimated prevalence of AMD is $11 \%$ in those aged 80 years and over (2), and 95\% of cases of legal blindness in this age group are caused by AMD.

Macrophages and neovascularization Macrophages have been shown to have both pro- and antiangiogenic effects following tissue injury in the eye, which suggests that their activity is carefully regulated in a complex manner, likely by local cytokines: on one hand, it has for instance been shown that macrophages are essential for removal of the hyaloid vessels; on the other hand, macrophages are necessary to 